\title{
Neural Correlates of Color-Selective Metacontrast in Human Early Retinotopic Areas
}

\author{
Kiyohiro Maeda, ${ }^{1,2}$ Hiroki Yamamoto, ${ }^{1}$ Masaki Fukunaga, ${ }^{3}$ Masahiro Umeda, ${ }^{3}$ Chuzo Tanaka, \\ and Yoshimichi Ejima ${ }^{5}$ \\ ${ }^{1}$ Graduate School of Human and Environmental Studies, Kyoto University, Kyoto; ${ }^{2}$ Imaging Technology Center, FUJIFILM, Kanagawa; \\ ${ }^{3}$ Departments of Medical Informatics and ${ }^{4}$ Neurosurgery, Meiji University of Integrative Medicine, Kyoto; and ${ }^{5}$ Kyoto Institute \\ of Technology, Kyoto, Japan
}

Submitted 14 October 2009; accepted in final form 20 July 2010

\begin{abstract}
Maeda K, Yamamoto H, Fukunaga M, Umeda M, Tanaka C, Ejima Y. Neural correlates of color-selective metacontrast in human early retinotopic areas. J Neurophysiol 104: 2291-2301, 2010. First published July 21, 2010; doi:10.1152/jn.00923.2009. Metacontrast is a visual illusion in which the visibility of a target stimulus is virtually lost when immediately followed by a nonoverlapping mask stimulus. For a colored target, metacontrast is color-selective, with target visibility markedly reduced when the mask and target are the same color, but only slightly reduced when the colors differ. This study investigated neural correlates of color-selective metacontrast for coneopponent red and green stimuli in the human V1, V2, and V3 using functional magnetic resonance imaging. Neural activity was suppressed when the target was rendered less visible by the same-colored mask, and the suppression was localized in the cortical region retinotopically representing the target, correlating with the perceptual topography of visibility/invisibility rather than the physical topography of the stimulus. Retinotopy-based group analysis found that activity suppression was statistically significant for V2 and V3 and that its localization to the target region was statistically significant for V2. These results suggest that retinotopic color representations in early visual areas, especially in V2, are closely linked to the visibility of color.
\end{abstract}

\section{N T R O D U C T I O N}

Metacontrast is a visual masking illusion in which the visibility of a briefly presented target stimulus is virtually lost when immediately followed by a nonoverlapping mask stimulus (Breitmeyer and Ogmen 2006). Interestingly, metacontrast is largely color-selective, peaking when the mask and target are the same color (Beer and MacLeod 2003; Bevan et al. 1970; von der Heydt et al. 1998). This study explored whether neural activity is suppressed in the human early retinotopic areas V1, $\mathrm{V} 2$, and V3 when color visibility of the target is reduced by color-selective metacontrast and further investigated whether the suppression is retinotopically localized to the cortical region representing the colored target.

This exploration is important for understanding the neural processes underlying color visibility. Metacontrast has been used as a tool to investigate mechanisms underlying visibility for achromatic patterns in several physiological studies, which have accumulated evidence for the involvement of early areas for visibility (Macknik 2006). However, applications to chromatic patterns have not previously been reported, thus leaving

Address for reprint requests and other correspondence: H. Yamamoto, Graduate School of Human and Environmental Studies, Kyoto Univ., Yoshidanihonmatsu-cho, Sakyo-ku, Kyoto 606-8501, Japan (E-mail: yamamoto @ cv.jinkan.kyoto-u.ac.jp). unresolved the issue of whether early areas are also related to the visibility of color. Of particular interest is whether the retinotopic representation of the colored target is suppressed when its visibility is reduced. In the luminance dimension, many neuroimaging studies on visual masking have found that spatial distribution of activity in the early retinotopic areas are topographically correlated with visibility rather than physical stimulus (Macknik and Haglund 1999; Zenger-Landolt and Heeger 2003). If we find such topographic representations of visibility for color-selective metacontrast, it would suggest close contribution of the early areas to visibility also in the color dimension.

In addition to the exploration of neural correlates of color visibility, this study aims to resolve an apparent discrepancy on metacontrast suppression of cortical target representation between nonhuman and human studies. Although cat and monkey single-unit studies on metacontrast have found activity suppression of V1 neurons retinotopically representing the target (Bridgeman 1975, 1980; Macknik and Livingstone 1998), a human neuroimaging study found no evidence of this phenomenon (Haynes et al. 2005). The present study sought to resolve this apparent discrepancy between the nonhuman and human studies.

To address these questions, we performed functional MRI (fMRI) experiments in which brain activity was directly compared between when a target was followed by a heterochromatic mask (target was highly visible) and when a target was followed by an isochromatic mask (target was less visible). We predicted that if color representations in a given retinotopic area are associated with color visibility, brain activity should be suppressed when the target is made less visible by the isochromatic mask, and this suppression should be localized in the cortical region retinotopically representing the target.

A key feature of our experimental strategy is that the stimulation paradigm was free from two major difficulties in isolating the presumptive local masking effects using fMRI. The first difficulty lies in the isolation of the target-related activity. In a typical metacontrast stimulus presentation, as a mask adjoins a target, fMRI signals evoked from the cortical region retinotopically representing the target would be contaminated by the mask-related signals, if "with mask" and "without mask" conditions were compared. Instead, we presented the mask consistently and controlled target visibility solely by color combination between the target and mask. The second difficulty lies in the isolation of the visibility-related activity. Metacontrast display often accompanies "split" apparent mo- 
tion in which the target is perceived to have split and jumped into the mask region, even if the target itself is invisible (Otto et al. 2006; Stoper and Banffy 1977). Such motion-related brain activity could thus contaminate the data when target visibility is experimentally controlled by changing the time interval between target and mask, as used in earlier studies (Bridgeman 1975; Haynes et al. 2005; Kondo and Komatsu 2000; Macknik and Livingstone 1998). Our paradigm using color selectivity of metacontrast allowed us to control target visibility without changing motion perception.

\section{METHODS}

\section{General}

SUBJECTS. Eight subjects, including three of the authors, participated in this study. Six of the eight subjects participated in the fMRI experiments, and three of these six also participated in the psychophysical experiments. The remaining two subjects participated in a subset of the psychophysical experiments. Subjects had no history of psychiatric or neurological diseases, and all provided written informed consent. Subjects were experienced psychophysical observers who had normal color vision as assessed by the Ishihara test and ND-100 hue test (Japan Color Research Institute, Saitama, Japan) and normal or correctedto-normal visual acuity. The study protocols were approved by the Human Studies Committee at each participating institution.

DISPLAY APPARATUS AND CALIBRATION. Visual stimuli were generated using a VersaPro VA10 personal computer (NEC, Tokyo, Japan) equipped with a VSG2/4 video card (Cambridge Research Systems, Rochester, Kent, UK). Stimuli were displayed on a rearprojection screen by a UP-1100 digital-light-processing (DLP) projector (PLUS Vision, Tokyo, Japan; $704 \times 443$ pixels; refresh rate, 62 $\mathrm{Hz}$ ). The viewing distance was $18 \mathrm{~cm}$. The display was colorimetrically calibrated using a PR-704 or PR-650 spectroradiometer (Photo Research, Chatsworth, CA) in accordance with the standard procedure (Brainard 1995; Cowan 1995).
VISUAL STIMULI. The metacontrast masking stimulation (Fig. $1 A$ ) comprised a briefly flashed red or green target stimulus followed by a mask stimulus. The mask color is the same as or opposite to that of the target. In the psychophysical and fMRI experiments described below, the strength of perceptual and neural masking was compared between the hetero- and isochromatic pairs. The target and mask were each displayed for $81 \mathrm{~ms}$, and stimulus onset asynchrony (SOA) of the two stimuli was $113 \mathrm{~ms}$. The target-mask sequence was followed by 306 ms of uniform background display. The target was an annular shape, and the mask comprised a circular shape and an annular shape sandwiching the target. Both stimuli were centered on the fixation point at the center of the screen, which subtended $40 \times 53^{\circ}$ of visual angle. The annular target was presented parafoveally with visual field eccentricities of $5.0-8.5^{\circ}$. The mask did not spatially overlap or share contours with the target, but sandwiched the target with $1.8^{\circ}$ gaps. The foveal mask and far-peripheral mask elements subtended $0-3.2^{\circ}$ and $10.3-17.6^{\circ}$ of visual angle, respectively.

Colors were specified using the MacLeod-Boynton chromaticity diagram (MacLeod and Boynton 1979), whose coordinates $[\mathrm{L} /(\mathrm{L}+$ $\mathrm{M}), \mathrm{S} /(\mathrm{L}+\mathrm{M})]$ represent relative long- $(\mathrm{L})$, middle- $(\mathrm{M})$, and short-wavelength-sensitive (S) cone excitations at a constant luminance level. Cone excitations were calculated from the Smith-Pokorny cone fundamentals (Smith and Pokorny 1975). All colors we used lay on the $\mathrm{L} /(\mathrm{L}+\mathrm{M})$ axis in the chromaticity diagram. Except for matching stimuli used in psychophysics, chromaticities and luminance were fixed as below. The target and mask were red [0.70, 1.14] or green $[0.62,1.14]$ on a gray background $[0.66,1.14]$. In cone contrast space, the opponent pair lies at exactly opposite points around the origin, minimizing color adaptation when the opponent pair was alternately and repeatedly presented in experiments. We added a $20 \%$ luminance increment to the colored target and mask to strongly activate neurons, as the majority of color neurons respond to combined chromatic and luminance contrast, rather than purely chromatic contrast (Johnson et al. 2001). The added luminance contrast should thus reinforce brain activity and should evoke clearer fMRI responses. If we had used isoluminant color stimuli, all of the resulting brain activity could have been attributed to the action of color mechanisms,
A
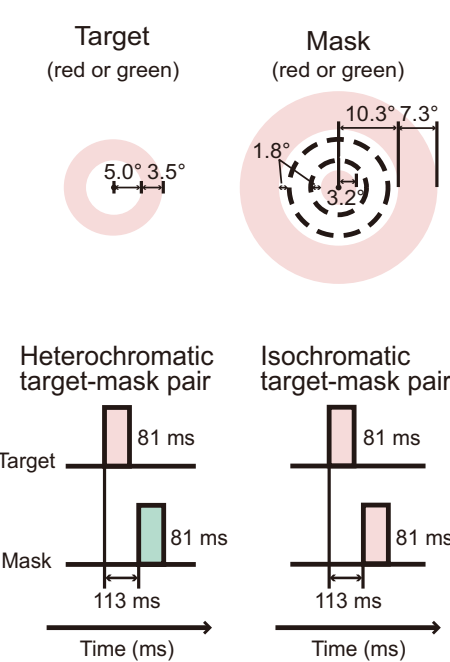

B
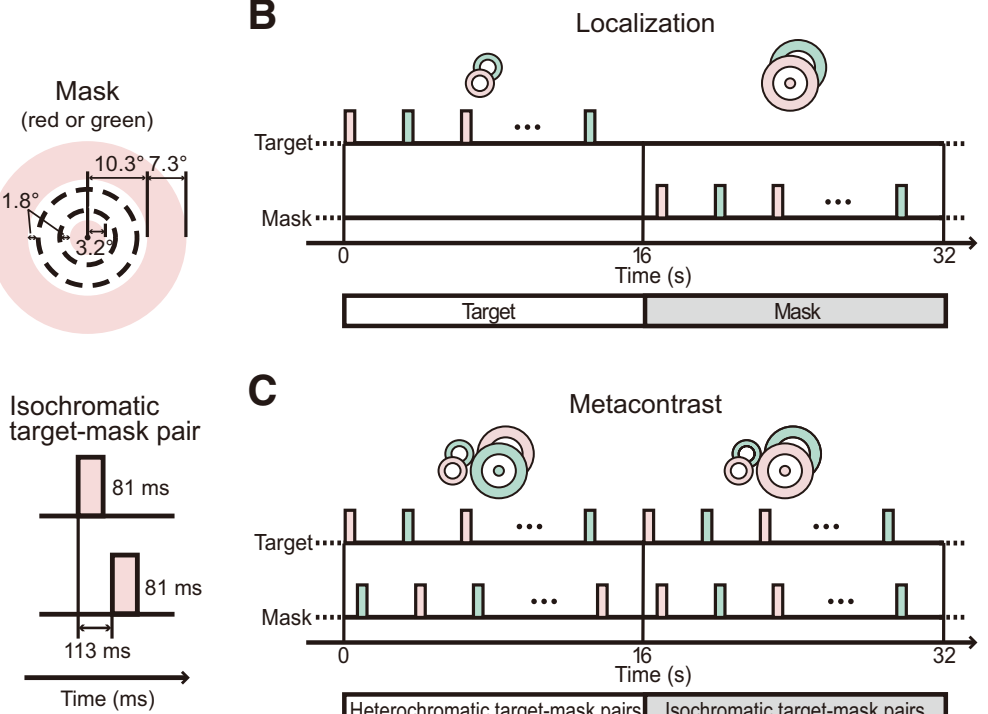

C

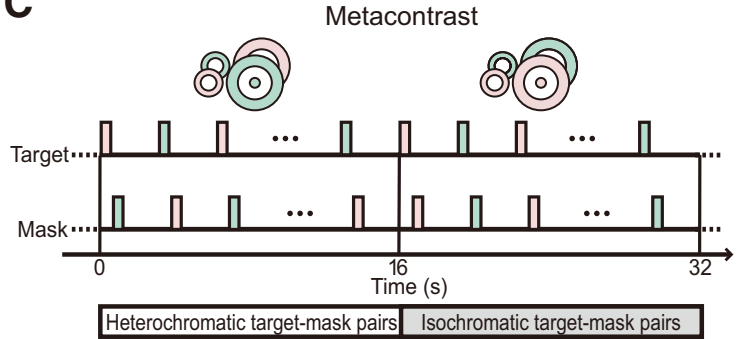

FIG. 1. Stimulus description. A: a single metacontrast stimulus comprising an annular target and a backward mask enclosing the target. The target and mask

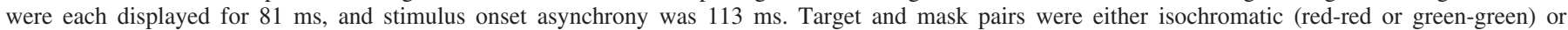

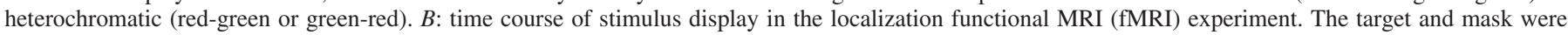

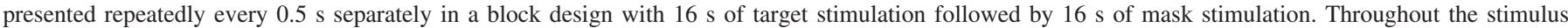

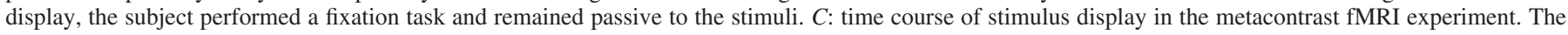

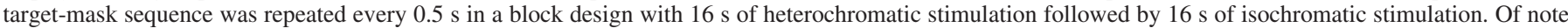


experiment. 
whereas the nonisoluminant colors would also activate luminance mechanisms. Thus our interpretation of the data requires some assumptions to estimate the relative contributions of the two mechanisms (see DISCUSSION). However, the use of isoluminance would lead to less activation and thereby make it harder to detect modulation in fMRI responses because the extent of modulation was expected to be small for our experimental design in which visual stimulation was constant between the experimental blocks at any retinal location (see METACONTRAST EXPERIMENT). We chose to show the evidence for response modulation as clearly as possible, using luminance contrast stimuli.

\section{Psychophysical experiments}

STIMULUS DISPLAY. Subjects sat upright in a dark room with their heads stabilized in a chin rest and viewed stimuli rear-projected on the screen by the DLP projector. The background luminance was $70 \mathrm{~cd} / \mathrm{m}^{2}$.

EXPERIMENTAL DESIGN. Psychophysical experiments were designed to confirm color selectivity in our metacontrast display by comparing the visibility of the target followed by the same-colored mask with that followed by the different-colored mask. Relative masking strength of the isochromatic and heterochromatic masks (Fig. 2) was measured using a two-alternative, temporal forcedchoice technique. Each trial comprised two intervals: one in which the heterochromatic target-mask pair was presented and one in which the isochromatic pair was presented. The order of intervals was randomized. Within a trial, the subject maintained fixation and pressed a button when they detected the character A, which appeared at the fixation point synchronized with the target flash with a probability of one third. After each trial, the subject indicated the interval in which the target had higher visibility. The independent variable was color strength of the test target of a heterochromatic pair, defined as excursion from the background gray with respect to both luminance $(\mathrm{L}+\mathrm{M})$ and chromatic $[\mathrm{L} /(\mathrm{L}$ $+\mathrm{M})$ ] dimensions, whereas the ratio (color angle) was kept constant. The color strength of the target followed by the heterochromatic mask was adjusted to match the visibility of the reference target followed by the isochromatic mask. The matching point was measured using psychophysical techniques (see Supplemental Methods for details) ${ }^{1}$ under three conditions, which differed with respect to the durations of the intervals. In the first condition, the target-mask sequence was presented only once in each 0.5 -s interval. In the second condition, the target-mask sequence followed by the blank background was repeated six times during each 3-s interval. From one repetition to the next, the color of the target and mask alternated between red and green to minimize color adaptation. In the third condition, the target-mask sequence was repeated 32 times in each 16-s interval, as in the fMRI experiments described below. Five subjects participated in the experiment of the first condition, three of whom had also participated in the fMRI experiments. Two of the three subjects participated in the experiments of the second and the third conditions.

In addition to the measurements of the relative visibility change between the isochromatic and heterochromatic conditions, absolute target visibility change was measured for each of the iso- and heterochromatic target-mask pairs. These absolute measurements were performed using a similar procedure to that of the relative measurement with the shortest intervals, with the exception that one of the two intervals contained the target alone, and its color strength was adjusted. Five subjects participated in this experiment.

We also examined the relative visibility of the mask stimulus in a similar way to the measurements of the target visibility, with the exception that color strength of the matching mask preceded by a heterochromatic target varied and the subject was instructed to indicate the interval in which the mask had higher visibility. Five subjects participated in experiments using the 0.5 -s interval condition, and two of them also participated in experiments using the 3 -s interval condition.
STATISTICS. For all psychophysical measurements, the statistical significance of visibility reduction was determined by a two-tailed $t$-test. The null hypothesis is that there is no significant difference in visibility between the test and reference target or mask; that is, matching color strength equals the color strength of the reference target or mask. $P<0.05$ was considered statistically significant.

\section{Functional MRI experiments}

IMAGE ACQUISITION. The MRI apparatus and image acquisition protocols have been described elsewhere (Ban et al. 2006; Yamamoto et al. 2008). Briefly, MRI was performed using a Signa 1.5-T clinical scanner (General Electric, Fairfield, CT). Structural images for anatomical registration were acquired using a T1-weighted three-dimensional fast spoiled gradient recalled echo pulse sequence (TR, $8.9 \mathrm{~ms}$; TE, $1.8 \mathrm{~ms}$; FA, $10^{\circ}$; FOV, $200 \times 200 \mathrm{~mm}$; matrix, $256 \times 256$; slice thickness, $1.4 \mathrm{~mm}$; axial 124 slices). Multiple functional scans were obtained while the subject viewed the visual stimuli, with a T2*weighted gradient echo echo planar imaging sequence (TR 2,000 ms; TE, $50.0 \mathrm{~ms}$; FA, $90^{\circ}$; FOV, $200 \times 200 \mathrm{~mm}$; matrix, $128 \times 128$; slice thickness, 3 or $4 \mathrm{~mm}$; 16 or 17 slices). For each scan, 101 functional images depicting blood oxygen level-dependent contrast (Ogawa et al. 1990) were collected from each slice. These slices included the occipital, posterior parietal, and temporal lobes, oriented roughly parallel with or perpendicular to the calcarine sulcus.

STIMULUS DISPLAY. Subjects were positioned in supine and viewed stimuli presented by the DLP projector via a surface reflection mirror. The original lens of the projector was replaced with an APO 170-500 $\mathrm{mm}$ zoom lens (Sigma, Kanagawa, Japan) set at 500-mm focal length to focus a small image on the distant screen. Background luminance was decreased from 96 to $67 \mathrm{~cd} / \mathrm{m}^{2}$ during the course of the study to produce high color contrast stimuli given the inevitable aging of the projector lamp. Head movement was minimized using a custom-made head fixation device.

LOCALIZATION EXPERIMENT. A set of two experiments was conducted to investigate the potential relationship between color visibility and cortical representations of the target stimulus. The first was localization of cortical regions retinotopically representing the target stimulus. Using a blocked design, the target alone was repeatedly presented for 81 ms every $500 \mathrm{~ms}$ in the first 16-s block; then, the mask alone was repeatedly presented for the same duration in the second 16-s block (Fig. $1 B)$. Stimulus color alternated between the opponent pair of red and green to minimize color adaptation. In each scanning run, the two alternating blocks were repeated six times after a 14-s rest interval. Subjects were instructed to passively view the color stimuli while maintaining fixation and to press a button, as in the psychophysical experiments, when the character A appeared at the fixation point. Each subject performed six to nine runs over 2-3 days.

METACONTRAST EXPERIMENT. The second experiment was a metacontrast experiment to investigate the cortical processes underlying color-selective metacontrast. The protocol of this experiment (Fig. $1 C$ ) was similar to that of the psychophysical experiment using the longest observation intervals. Using a blocked design, heterochromatic target-mask sequences were repeatedly presented every $500 \mathrm{~ms}$ in the first 16-s block; then, isochromatic target-mask sequences were repeatedly presented in the second 16-s block. Notably, neither the target nor mask underwent any physical change across blocks throughout the run. In each scanning run, the two alternating blocks were repeated six times after a 14-s rest interval. Again, subjects were instructed to passively view the color stimuli while carrying out the fixation task. Each subject performed 18-27 runs over 2-3 days.

IDENTIFICATION OF VISUAL AREAS. Retinotopic visual areas V1v(d), $\mathrm{V} 2 \mathrm{v}(\mathrm{d})$, and $\mathrm{V} 3 \mathrm{v}(\mathrm{d})$ (Fig. S1) were localized on the cortical surface of each subject using standard retinotopic mapping procedures (Engel et al. 1994; Sereno et al. 1995; Yamamoto et al. 2008). Briefly, the 


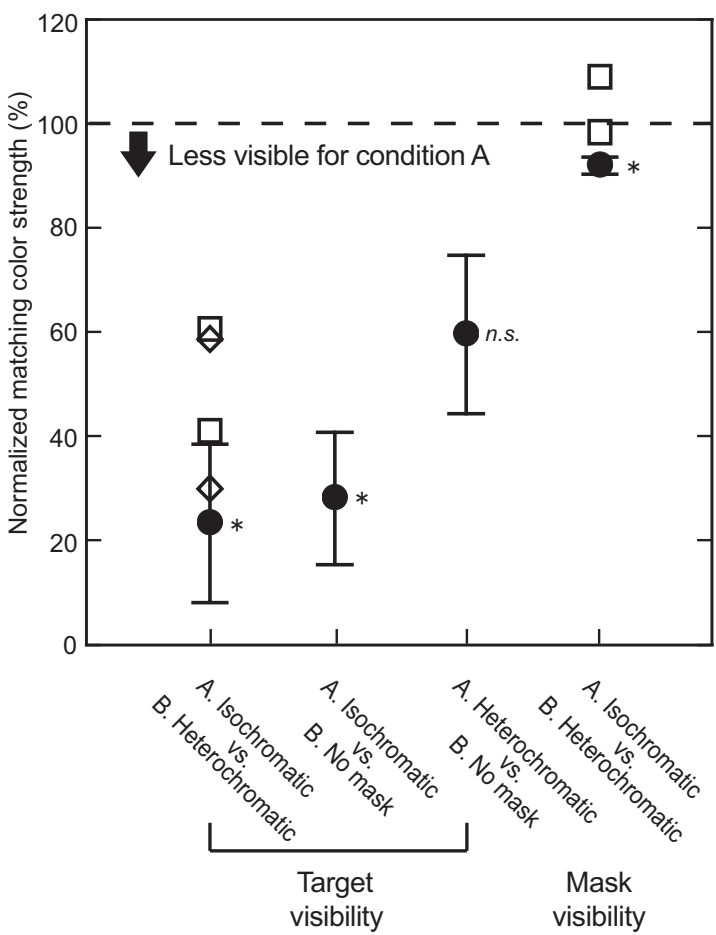

FIG. 2. Magnitude of visibility reduction for target or mask stimuli measured by matching experiments. From left to right, matching data from 3 experiments for target visibility and 1 experiment for mask visibility are shown. The horizontal dashed line $(100 \%)$ indicates color strength of the reference target or mask in "condition A" described in the abscissa. Each plot represents matching color strength of the test target or mask in "condition B," giving the same visibility as the reference stimulus. If no difference in visibility is present, matching color strength of $100 \%$ is expected, and a value $<100 \%$ indicates a reduction in visibility for condition A. For each of the 4 experiments, the average matching point (solid circles) across 5 subjects when the target-mask sequence was presented once is plotted. The error bars indicate $\pm \mathrm{SE}$ across subjects. The open symbols for matching experiments between the isochromatic vs. heterochromatic pairs represent individual matching points from 2 subjects when the test-mask sequence was repeated 6 (open squares) or 32 times (open diamonds). Asterisks indicate significant visibility reduction.

cortical surface (Fig. S1A) and its hypersmoothed version (Fig. S1B) were reconstructed from a high-contrast structural volume scanned once before the experimental sessions. Surface regions delimiting visual areas were determined by phase-encoded retinotopic mapping methods allowing visualization of the polar angle and eccentricity components of the retinotopic map.

\section{Functional MRI data analysis}

fMRI data analysis was performed using in-house software (Yamamoto et al. 2002, 2008).

fMRI SIGNAL SAMPLING AND PREPROCESSING. After corrections for head motion (Woods et al. 1998) and time misalignments between slices (McKeown et al. 1998), functional images were registered to the cortical surface of each subject. The fMRI data were sampled independently from each visual area. The sampled fMRI signal was processed as follows. First, the first portion of time points up to the first two blocks was discarded to avoid transient effects. Second, classical decomposition (Brockwell and Davis 1991) was applied to the data to remove components slower than the block alternation frequency (1/32 s). Third, the data were converted to percent signal change by dividing by the mean signal intensity of the time series.

ISOECCENTRICITY ANALYSIS. The fMRI responses from localization and metacontrast experiments were analyzed spatially within each visual area, as a function of cortical geodesic distance along which retinotopic representation of visual field eccentricity shifted from the fovea to the periphery, using a technique described elsewhere (Ban et al. 2006; Yamamoto et al. 2008). First, for each node within the cortical mesh of a visual area, the geodesic distance from the peripheral $16^{\circ}$ contour was computed using Dijkstra's algorithm (Dijkstra 1959; Wandell et al. 2000). Second, using the distance information, the cortical mesh was divided into isoeccentricity bands ( $3 \mathrm{~mm}$ width with $50 \%$ overlap) from posterior to anterior cortex, so that the eccentricity representation changed from fovea to periphery. Third, fMRI responses were averaged within each isoeccentricity band. Finally, responses for different eccentricities were further averaged across repeated scanning runs and stimulus cycles. Averaged spatiotemporal response was displayed as a pseudo color image (Figs. 3 and 4; Figs. S2-S7).

RETINOTOPIC MORPHING. For each visual area, spatiotemporal fMRI responses from different subjects were combined into a standardized spatial response (Fig. 5) using a retinotopic morphing method (Yamamoto et al. 2008) that took into account individual, hemispherical, and dorsoventral differences in retinotopic organization. First, amplitudes of responses for different eccentricities were estimated by least-squares fitting of a model waveform, yielding a plot of response amplitude as a function of representing eccentricity. The waveform was obtained by convolution of a boxcar (ON-OFF) function representing heterochromatic (ON) and isochromatic blocks (OFF) with a hemodynamic response function (Boynton et al. 1996). Amplitude was thus positive when the isochromatic block showed lower activity than the heterochromatic block, indicating relative response suppression for the isochromatic stimuli. Second, after localizing the cortical region representing the target or mask (see REGION OF INTEREST ANALYSIS), we estimated the cortical distance of the target, inner, and outer mask regions and mean values for different subjects, hemispheres, and dorsoventral positions. Third, the abscissa of the eccentricity-amplitude plot was piecewise linearly scaled such that each part of the plot (corresponding to the target, inner, and outer parts of the mask) matched the mean value in distance. Finally, plot data were averaged across hemispheres, dorsoventral parts and subjects.

REGION OF INTEREST ANALYSIS. In region of interest (ROI) analyses, we analyzed fMRI responses separately for target- and maskrepresenting cortical regions as follows. First, for each visual area, the cortical region retinotopically representing the target was localized as the region in which fMRI response was phase-locked to presentation of the target in the localization experiment. Cortical regions representing inner and outer parts of the mask were likewise localized as regions in which response was phase-locked to mask presentation. Second, fMRI time series obtained in the metacontrast experiment were averaged across eccentricities within each of the target- and mask-representing regions and then across hemispheres, dorsoventral positions, and stimulus cycles. Third, the target and mask time series were separately averaged across subjects (Fig. 6, $A$ and $B$ ). Fourth, amplitude for the averaged time-series was computed by fitting the model response as described in Retinotopic morphing (Yamamoto et al. 2008). The final amplitude represents our estimate of response for each of the target and mask regions (Fig. 6C).

RESPONSE LOCALIZATION INDEX. In the final step of our analyses (Fig. 6D), we evaluated the locality of response suppression within each visual area by calculating the response localization index for each subject from response amplitudes of target and mask time-series by the following equation

$$
\text { Localization index }=\frac{\text { Target amplitude }- \text { Mask amplitude }}{\mid \text { Target amplitude }|+| \text { Mask amplitude } \mid}
$$

This index takes a maximum value of 1 when target amplitude is positive and mask amplitude is negative or zero, indicating that suppression is perfectly localized to the target region. Conversely, a minimum value of -1 indicates that suppression is perfectly localized to the mask region. A 
A

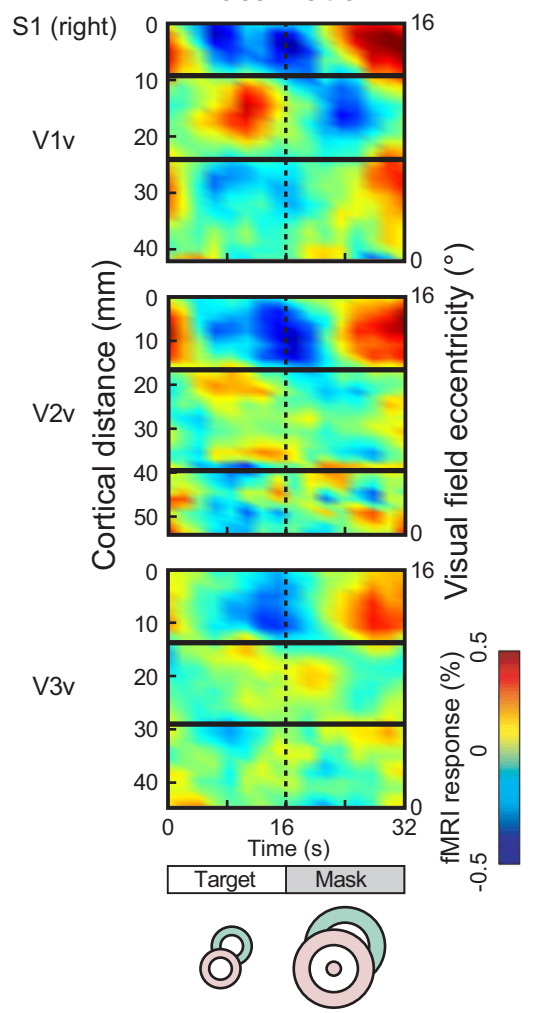

B

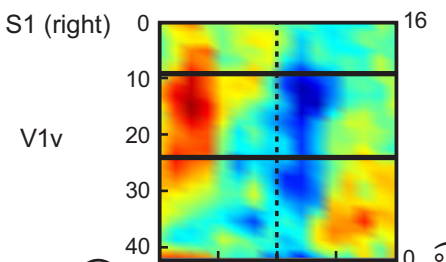

है
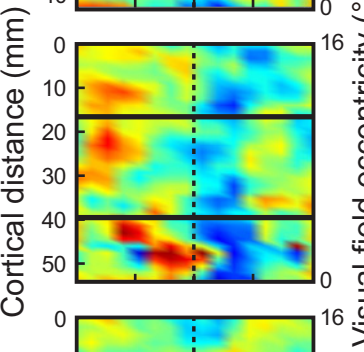

V3v

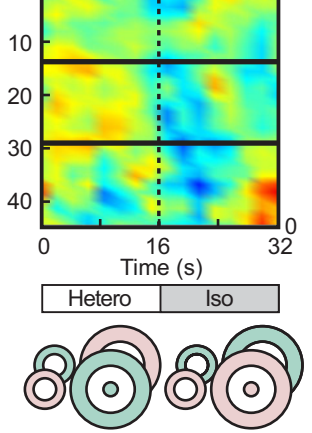

A

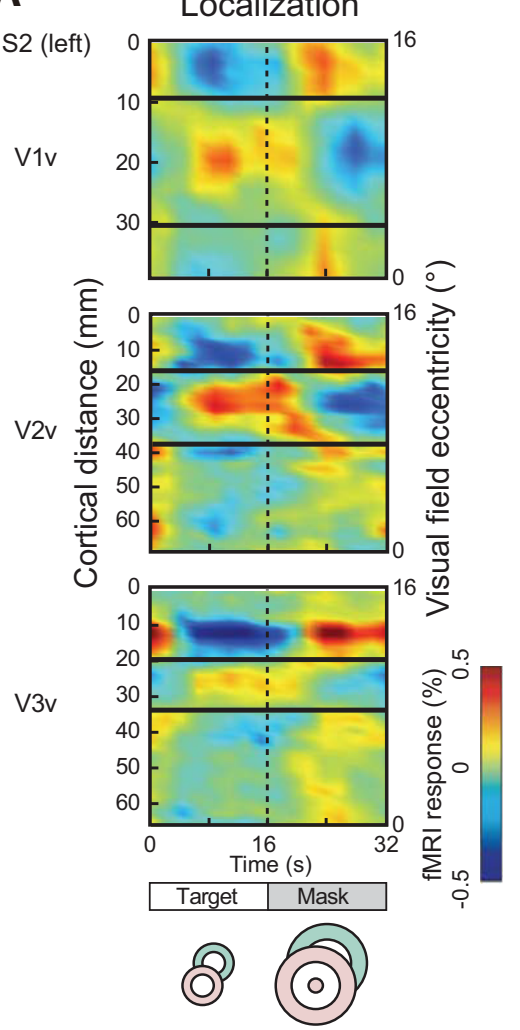

B

S2 (left)

V1v

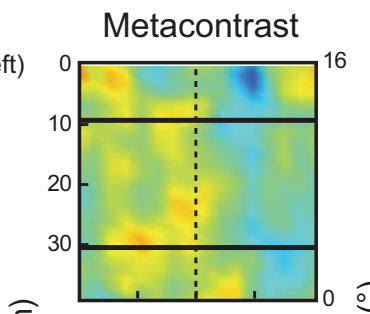

○

है

V3v

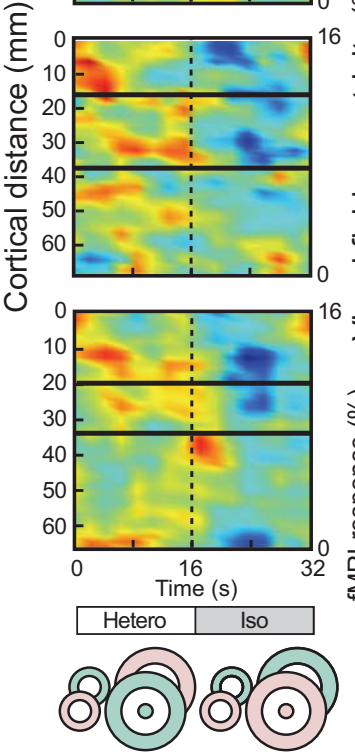

FIG. 3. Spatiotemporal fMRI responses in areas V1v, V2v, and V3v (subject S1, right hemisphere). For each area, fMRI responses from the cortical region with the same preferred eccentricity were averaged using isoeccentricity analysis. The result is shown as a pseudocolor image, in which individual rows represent individual fMRI time series at different preferred eccentricities from the fovea to the periphery and corresponding cortical distances. Peripheral $16^{\circ}$ corresponds to 0 cortical distance. A: spatiotemporal brain activity in the localization experiment. Within each image, the cortical region retinotopically representing the target was identified as a region of interest (ROI) from phase reversals of the fMRI time series (the region bordered by the black lines). $B$ : spatiotemporal brain activity in the metacontrast experiment, overlaid with the ROI (bordered by the black lines). value of 0 indicates no difference in response between the two regions. This robust localization metric can accommodate a wide range of intersubject variability because it compensates for differences in neural sensitivity to the present target-mask stimulation.
STATISTICS. To determine the statistical significance of response suppression in fMRI time series data averaged across subjects, we performed a regression $t$-test (Draper and Smith 1998). In this test, we used the effective degrees of freedom for fMRI time series analysis 
A
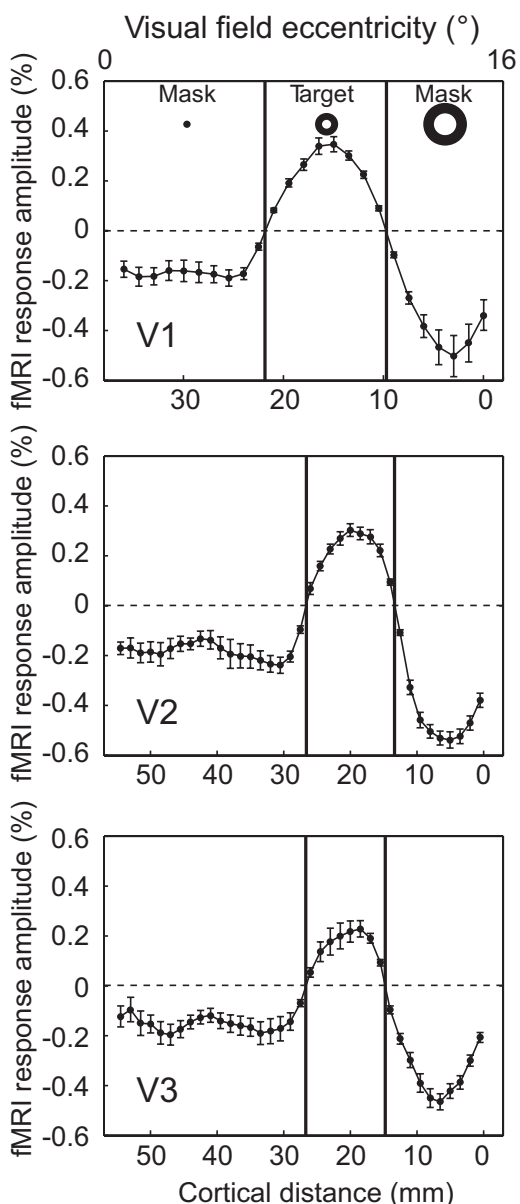

B
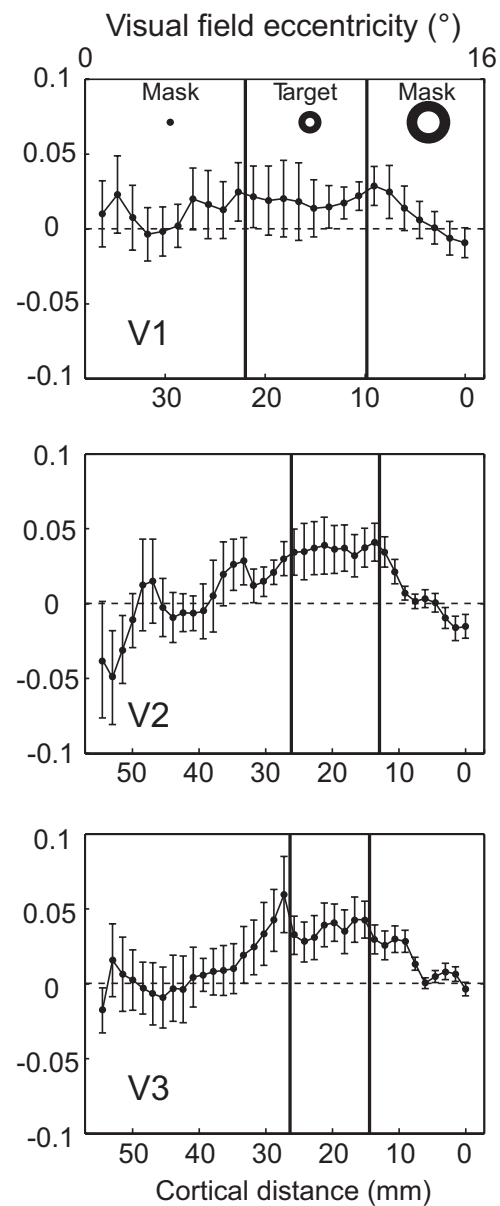

FIG. 5. Average spatial fMRI responses in V1, V2, and V3 of 6 subjects. For each of V1, V2, and V3, fMRI response amplitude (modulation depth of the response between experimental blocks) averaged across hemispheres, dorsoventral parts, and subjects is plotted against preferred eccentricity from the fovea to the periphery and corresponding cortical distances. Peripheral $16^{\circ}$ corresponds to 0 cortical distance. The amplitude was defined as positive when the response during the 1st block was higher than the 2nd and as negative when the opposite was observed. Error bars indicate $\pm \mathrm{SE}$ across subjects. $A$ : results of the localization experiment: target vs. mask. $B$ : results of the metacontrast experiment: heterochromatic target-mask pair vs. isochromatic target-mask pair.
(Worsley and Friston 1995). The null hypothesis is that there is no significant response suppression for the isochromatic block; that is, mean amplitude is zero. To determine the significance of the response localization effect, we used the localization index and performed a two-tailed $t$-test across subjects. The null hypothesis is that there is no localization effect; that is, the mean index value is zero. For these tests, $P<0.05$ was considered statistically significant.

Preliminary results of this study were presented in abstract form at the 9th International Conference on Functional Mapping of the $\mathrm{Hu}-$ man Brain held June 2003, New York, NY, and at the Optical Society of America Fall Vision Meeting held October 2005, Tucson, AZ.

\section{RES ULT S}

\section{Psychophysical experiments}

In psychophysical experiments, we confirmed that subjects experienced reliable color-selective metacontrast when using our stimulus configuration, in which a colored annular target stimulus was followed by a colored mask stimulus that surrounded the target (Fig. 1A). Color visibility for the display was examined in a series of matching experiments using a two-alternative, temporal forced-choice procedure, during which the subject's attention was diverted away from the parafoveal target by imposing a foveal task.

In the first series of experiments, visibility was compared between when a target was followed by an isochromatic mask and when it was followed by a heterochromatic mask for both red and green targets. Color strength (combined luminance and color, see METHODS) of the target followed by the heterochromatic mask was adjusted to match the visibility of the reference target followed by the isochromatic mask. This direct comparison required lower matching color strength for the target followed by the heterochromatic mask (Fig. 2, left-most col$u m n$, solid circle; $P=0.007,2$-tailed $t$-test, $\mathrm{df}=4)$, indicating greater visibility reduction for the isochromatic pair, and thus color-selective metacontrast. This metacontrast effect persisted when the target-mask sequence was repeated multiple times (Fig. 2, left-most column, open symbols), as in the fMRI experiments described below.

Next, visibility for each of the isochromatic or heterochromatic pairs was compared with that for the target presented alone (Fig. 2, 2nd and 3rd columns). This comparison required lower matching color strength of the unmasked target for the isochromatic pair $(P=0.005,2$-tailed $t$-test, $\mathrm{df}=4)$, whereas the difference was not significant for the heterochromatic pair $(P=0.056)$. The results indicate that the difference obtained in the direct comparison was not just relative, confirming an absolute reduction in target visibility for the isochromatic pair.

Finally, we examined the visibility of the mask stimulus using a similar experimental design to that used in the first experiment, but in this context, the color strength of the heterochromatic mask stimulus was varied instead of the target stimulus. Mask visibility was lower for isochromatic pairs than 
A
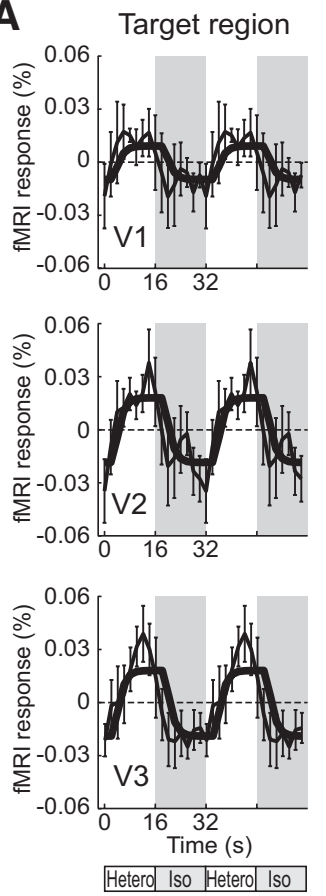
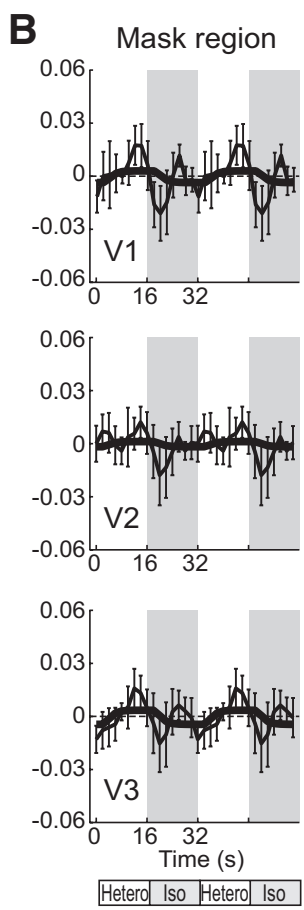

C
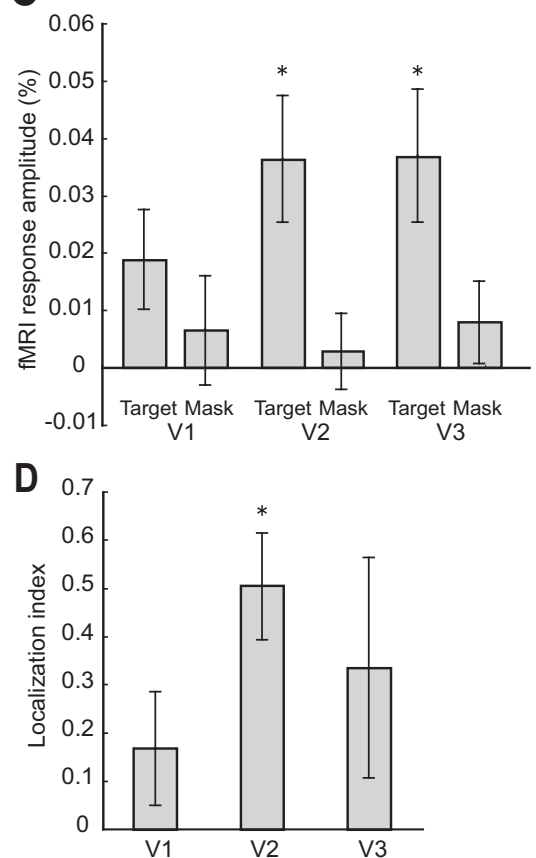

FIG. 6. Average fMRI response time series (thin lines) observed in the metacontrast experiment for the target-representing region $(A)$ and mask-representing region $(B)$, overlaid with the fitted model response (thick lines). Data during alternating hetero- and isochromatic blocks are plotted twice to better display the cyclic response pattern. Error bars indicate $\pm \mathrm{SE}$ across subjects. $C$ : response amplitudes of the fMRI time series for target- and mask-representing regions shown in $A$ and $B$, respectively. Positive values indicate a lower response when a target was followed by an isochromatic mask than when followed by a heterochromatic mask. Error bars indicate $\pm \mathrm{SE}$ of the regression coefficient. Asterisks indicate significant response suppression for the isochromatic condition. $D$ : response localization index for each visual area. The index takes a maximum value of 1 when response suppression occurred only for the target region but not for the mask region and a value of 0 when there was no localization effect. Error bars indicate \pm SE across subjects. Asterisk indicates significant response localization. for heterochromatic pairs (Fig. 2, right-most column; $P=$ 0.008 , 2-tailed $t$-test, df $=4$ ), but the magnitude of this reduction was much smaller than that for the target $(P=0.013$, 2 -tailed paired $t$-test, $\mathrm{df}=4)$. This reduction was not found when the target-mask sequence was repeatedly presented six times (Fig. 2, right-most column, open squares).

Altogether, a large reduction in color visibility clearly occurred only for the isochromatic pair within the visual field in which the target was presented.

\section{Functional MRI experiments}

LOCALIZATION EXPERIMENT. First, we localized cortical regions retinotopically representing the target in visual areas V1, V2, and V3 identified on the individual cortical surface. Specifically, we compared fMRI activity evoked by the target with that evoked by the mask, using a block paradigm. Subjects viewed only the target repeatedly flashed in one 16-s block and only the mask repeatedly flashed in another 16-s block (Fig. $1 B$ ). If a visual area was to have separate topographic representations for the target or mask, and if such representations were mutually exclusive, the direct comparison paradigm would reveal separate cortical regions, each of which would show a response increase when the associated stimulus appears.

Spatiotemporal response patterns in area V1 followed the prediction detailed above (Figs. $3 A$ and $4 A$; Figs. S2A-S7A). The observed response distribution showed a characteristic checkerboard pattern that correlated strongly with spatiotemporal modulation of the stimulus display. When the target annulus was presented at an intermediate eccentricity in the visual field, fMRI responses in the intermediate part of the cortical surface (bordered by 2 horizontal black lines in Figs. $3 A$ and $4 A$; Figs. S2A-S7A) increased with a hemodynamic delay of $\sim 4 \mathrm{~s}$. In contrast, when the mask was presented at the foveal and peripheral eccentricity, responses in the posterior and anterior parts of the cortical surface increased. The observed response pattern thus indicates that V1 had separate retinotopic representations for the target and mask. These retinotopic representations were observed for all six subjects. Very similarly to $\mathrm{V} 1$, we identified clear retinotopic representations of the target and mask in V2 and V3 (Figs. $3 A$ and $4 A$; Figs. S2A-S7A) for all subjects.

There was some variability in the spatiotemporal responses. For example, in some samples (e.g., Fig. S3A, V1d), response to the foveal part of the mask stimulus was weaker than to the peripheral part, whereas in other samples (e.g., Fig. $3 A, \mathrm{~V} 1 \mathrm{v}$ ), response to the foveal mask was comparable to the peripheral mask.

METACONTRAST EXPERIMENT. Second, we directly compared fMRI activity when a target was followed by a heterochromatic versus an isochromatic mask, using a block design to investigate neural correlates of color-selective metacontrast. Stimulus configuration and experimental design (Fig. 1, $A$ and $C$ ) were the same as those used in the psychophysical experiment, and the longest observation intervals were used. Subjects performed the foveal task as in the psychophysical experiment but did not perform the forced-choice task for target visibility.

Spatiotemporal response patterns in areas V1, V2, and V3 showed clear activity modulation depending on whether the target and mask were hetero- or isochromatic (Figs. $3 B$ and $4 B$; Figs. S2B-S6B; but modulation was unclear for 1 subject, Fig. $\mathrm{S} 7 B$ ). Specifically, the time course of activity modulation correlated positively with that of target perception: relative brain activity was increased when the target was highly visible in the heterochromatic block but was decreased or suppressed when the target was less visible in the isochromatic block. Importantly, although no physical modulation was present at each point of the stimulus across blocks, brain activity was modulated. 
Closer comparison of the response patterns in the metacontrast experiment (Figs. $3 B$ and $4 B$; Figs. S $2 B-\mathrm{S} 6 B$ ) with those for the localization experiment (Figs. $3 A$ and $4 A$; Figs. S2A$\mathrm{S} 6 A$ ) shows that response suppression in the isochromatic block tended to be strong in the vicinity of the cortical region retinotopically representing the target (bordered by two horizontal black lines). Typical examples are the data from $\mathrm{V} 1 \mathrm{v}$ in Fig. $3 B$ and V2v in Fig. $4 B$. In these data, strong suppression (dark red-to-blue transition between the hetero- and isochromatic blocks) was located in the vicinity of the target region. Such instances amount to 9, 12, and 12 cases in V1, V2, and V3, respectively, of 24 samples (6 subjects $\times 2$ hemispheres $\times$ 2 dorsoventral areas). Notably, in some cases, retinotopic suppression was biased toward the region corresponding to the inner and/or outer borders of the target. For example, in V2v in Fig. $4 B$, strong suppression in the isochromatic block was observed around both borders of the target region (indicated by horizontal black lines).

Locality was further investigated by averaging response amplitudes across subjects with preserving individual retinotopic organization (see METHODS), thereby enhancing sensitivity to retinotopic suppression. The results are shown in Fig. $5 B$ next to the results for the localization experiment (Fig. 5A), in which positive amplitudes indicate the relative response suppression by the color-selective metacontrast. Group-averaged data for V2 and V3 confirm that suppression occurred mainly in target-representing cortical regions with some bias favoring the target borders defined as the zero crossing points in the plots in Fig. 5A, whereas data for V1 showed less locality.

This suppression, however, was not totally confined to the target-representing region, but decreased gradually outside the target region into the mask region. The extent of spread was comparable to that found in $\mathrm{V} 1$ and $\mathrm{V} 2$ for responses to luminance and color edges (Cornelissen et al. 2006). The spread would thus not necessarily imply suppression of maskrelated activity.

Figure 6 compares the averaged fMRI time series of the target (Fig. 6A) and mask regions (Fig. 6B), which are overlaid with the fitted model response (see METHODS). It shows activity suppression during the isochromatic block compared with during the heterochromatic block for the target region, whereas it shows much less suppression for the mask region. The response amplitude (Fig. $6 C$ ) for the target region was significantly above zero for $\mathrm{V} 2(P=0.015$, regression 2-tailed $t$-test, $\mathrm{df}=6.45)$ and V3 $(P=0.016)$, indicating significant suppression, whereas it was not for V1 $(P=0.069)$. In contrast, no significant suppression was found for the mask region in any of these areas (V1: $P=0.511, \mathrm{~V} 2: P=0.676, \mathrm{~V} 3: P=0.301)$.

Finally, we evaluated the response localization to the target region by computing the localization index (see METHoDs). The index has a positive value when the target amplitude is larger than the mask amplitude and a maximum value of 1 for perfect localization (suppression for the target region only). The indices averaged across subjects took positive values for all of the areas (Fig. 6D), indicating localized suppression to the target region. The localization effect was statistically significant for V2 $(P=0.006$, 2-tailed $t$-test, $\mathrm{df}=5)$, whereas it was not for $\mathrm{V} 1(P=$ $0.213)$ or V3 $(P=0.200)$.

\section{I S C U S S I O N}

This study found that neural activity correlated with perception of color-selective metacontrast in human V1, V2, and V3, in that 1 ) neural activity was suppressed when the target was rendered less visible with the isochromatic mask compared with high visibility with the heterochromatic mask, and 2) response suppression was localized in the cortical region retinotopically representing the target. Retinotopy-based group analysis found statistically significant activity suppression in V2 and V3 and statistically significantly localized suppression to the target region in $\mathrm{V} 2$.

\section{Methodological advantages and limitations}

A key feature of the present stimulation paradigm was that the stimulus was modulated only in color combination between the target and mask, but no physical modulation was present at any point of the stimulus over hetero- and isochromatic targetmask conditions between which brain activity was compared (Fig. 1C). In other words, no modulation in stimulus existed between the two conditions for any point in the retina, which was stimulated by repeatedly alternating red and green flashes of light. This equality in stimulation is a critical point for the present purpose of examining the effects of color combination between metacontrast target-mask pairs, because resulting modulations in perception or brain activity cannot be attributed to changes in stimulus. Of particular note is that the present stimulation involved no change in temporal interval or SOA between the target and mask. This feature is different from many earlier physiological studies on metacontrast, which changed SOA to modulate visibility (e.g., Bridgeman 1975). Our stimulation method minimizes undesirable effects related to apparent motion perception, which often accompanies metacontrast (Otto et al. 2006; Stoper and Banffy 1977). Changing SOA would have considerably modulated the percept of motion because apparent motion is highly dependent on SOA. It would have therefore caused motion-related brain activity, which may contaminate the visibility-related activity of interest. Importantly, for these stimuli, the percept of motion was unlikely to depend on whether the target and mask had the same or different colors. Kolers and Green (1984) found no difference in motion perception between hetero- and isochromatic target-mask pairs with similar spatial and temporal parameters to ours. Indeed, our subjects reported no difference in motion perception between the stimulus pairs.

Despite this advantage in isolating visibility-related activity, our paradigm has a limitation in that it only identifies relative differences in brain activity between hetero- and isochromatic conditions and absolute changes in activity from when the target was presented alone in the absence of a mask are not indicated. Consequently, this method cannot distinguish response suppression in one condition from response enhancement in the other condition. However, it is parsimonious to suppose that the observed modulation was caused by response suppression in the isochromatic condition rather than response enhancement in the heterochromatic condition, assuming that changes in fMRI signal is corresponding to changes in visibility. This interpretation is consistent with the results of absolute measurements showing that metacontrast visibility reduction accompanies parallel activity suppression of electrophysiolog- 
ical (Bridgeman 1980; Macknik and Livingstone 1998) and fMRI signals (Haynes et al. 2005).

\section{Neural correlates of color visibility}

The present spatiotemporal activity map (Figs. $3 B$ and $4 B$; Figs. S2 $B-\mathrm{S} 7 B$ ) and the subject-average response (Figs. $5 B$ and 6) during metacontrast show target-specific activity suppression topographically mirroring reduced visibility (Fig. 2). This suggests that neural activity in V1, V2, and V3 does not necessarily correlate with physical topography of the stimulus presence/absence but rather with perceptual topography of the stimulus visibility/invisibility. This is consistent with the results of earlier electrophysiological studies, in which responses of V1 neurons representing the target location were found to be suppressed by a metacontrast display (Bridgeman 1975, 1980; Macknik and Livingstone 1998). However, a prior fMRI study in humans failed to find such retinotopic suppression in V1 for stimuli in which the masks adjoined the small $\left(0.8^{\circ}\right)$ targets (Haynes et al. 2005). In that case, brain activity evoked by the masks might have overlapped target activity because of the limited spatial resolution of fMRI. This contamination is crucial when the amount of suppression is relatively small, as in the present study. The present larger $\left(3.5^{\circ}\right)$ target and nonadjoining mask, and the retinotopic morphing analysis (see METHODS) to improve signal-to-noise ratio may have contributed to isolated target-related responses. For not metacontrast but a combined forward and backward masking, called the standing wave of invisibility (SWI) (Macknik and Livingstone 1998), evidence of retinotopic suppression has been established in other human fMRI (Huang et al. 2006; Tse et al. 2005) and monkey optical imaging (Macknik and Haglund 1999) studies. The present results provide the first human data showing retinotopic suppression for metacontrast.

The finding of neural correlates of color-selective metacontrast in V1, V2, and V3 suggests that color representations in these areas are closely linked to the visibility of color, providing strong evidence against the view that the roles of the early areas are limited to preprocessing and relaying afferent color signals to a higher area in the ventral occipital cortex, which is thought to be essential for color perception (Bartels and Zeki 2000; Tootell et al. 2003; Wade et al. 2008). The response suppression correlated with visibility reduction was markedly robust in V2 (Fig. 6), suggesting that V2 contributes more to visibility in the color dimension than V1. For the luminance dimension, several recent human neuroimaging studies have found evidence for the contribution of V1 to visibility, using near-threshold contrast stimuli (Ress and Heeger 2003) or surround masking stimuli (Ejima et al. 2007; Ohtani et al. 2002; Williams et al. 2003; Zenger-Landolt and Heeger 2003). This study adds new evidence for substantial contributions of $\mathrm{V} 1, \mathrm{~V} 2$, and V3 to visibility in the color dimension.

We should note that the target stimulus in this study differed from the background in both luminance and color. The observed response suppression may thus be related to the processes underlying visibility not only for color but also for luminance. When comparing the target color component with the luminance component in cone contrast units, the color component was 2.2 times smaller than the luminance component. Consequently, when we assume that human V1 has about 2-6 times greater sensitivity for color than for luminance
(Engel et al. 1997; Liu and Wandell 2005; Mullen et al. 2007, 2008; Parkes et al. 2009), $\sim 50-70 \%$ of the observed neural suppression would reflect the color response, disregarding the fact that the relative sensitivity to color and luminance depends on spatial and temporal aspects of stimuli. Apart from the degree of inherent color contribution to suppression, the suppression is clearly a consequence of the action of a color mechanism, because our target and mask were equiluminant.

\section{Implications for cortical color representations}

We speculate that color-selective response suppression may reflect the actions of two separate, unipolar channels for cone-opponent red or green signals. In the red channel, a red mask signal interferes with red target signals, whereas the green mask signal does not enter the red channel and has no influence on the red target signal. Such unipolar red and green mechanisms with intrachannel suppression have been found in psychophysical studies of simultaneous color contrast (Smith and Pokorny 1996), color noise-masking (Sankeralli and Mullen 2001), SWI (McKeefry et al. 2005), and metacontrast (Beer and MacLeod 2003; Bevan et al. 1970; Cavonius and Reeves 1983; von der Heydt et al. 1998; Yellott and Wandell 1976). The unipolar mechanisms are also consistent with the model of cortical color signal transformation in which subcortical bipolar opponent signals are half-wave rectified by cortical neurons with little spontaneous activity (De Valois and De Valois 1993; Solomon and Lennie 2005). Although unipolar mechanisms for color are likely underlying mechanisms, they are not necessarily pure chromatic mechanisms because the observed activity was evoked by the stimulus with a luminance contrast. However, underlying mechanisms must allow discrimination of two equiluminant colors, those of the target and mask.

Recently, two fMRI studies have found unipolar representations for colors by applying multivoxel pattern classification techniques to brain activity for simple color stimulation (Brouwer and Heeger 2009; Parkes et al. 2009). Other fMRI measurements, which analyzed the mean response by visual area, have shown and characterized cone opponency in V1 and V2 but found no evidence of unipolar representations for simple stimulus display (Engel et al. 1997; Liu and Wandell 2005; Mullen et al. 2007, 2008; Wandell et al. 1999). Taking advantage of metacontrast display, the present study was able to find evidence for unipolar representations by a conventional analysis of mean response.

The observed differences in activity suppression between V2 and V1 (Fig. 6) may have important implications in terms of cortical color representations, as well as their relative contribution to color visibility. The difference seems to indicate that unipolar representations are more dominant in V2 than in V1. This interpretation is consistent with the results from an optical recording study showing highly color-selective representations in V2 (Xiao et al. 2003). Such color representations are likely to be related to neurons found in monkey V2 and V1, which show narrower color tuning than that predicted from a linear combination of cone outputs, like the presumed unipolar channels (De Valois et al. 2000; Hanazawa et al. 2000; Kiper et al. 1997; Lennie et al. 1990; Wachtler et al. 2003). When comparing populations of the nonlinear neurons between $\mathrm{V} 2$ and $\mathrm{V} 1, \sim 35 \%$ of V2 color-selective neurons are narrowly tuned 
(Kiper et al. 1997), whereas for V1, the reported population of narrowly tuned neurons varies between studies from $\sim 2 \%$ (Lennie et al. 1990) to $\sim 50 \%$ (De Valois et al. 2000) of investigated neurons.

This study took advantage of color similarity dependence of metacontrast to characterize a relationship between cortical color representations and color visibility. Such similarity dependence has also been reported for luminance polarity (Becker and Anstis 2004). Furthermore, metacontrast masking depends on global or high-level shape similarities (Uttal 1970). These data lead us to hypothesize that multiple-channel structures with intrachannel suppression, in which each channel represents within-category information, underlie metacontrast not only for color but for other visual features. Elaboration and extension of the present paradigm to a variety of colors, shapes, and conjunctions of these may offer a promising way to show how many and what kind of categories are represented in the visual system.

\section{A C K N O W LE D G MENTS}

We thank T. Matsuno, who ran a pilot version of this study; A. Maeda for assistance in data gathering; and Y. Ohtani, J. Saiki, S. Takahama, H. Ban, N. Hagura, and H. Yamashiro for helpful comments on the manuscript. In addition, we thank H. Ikoma, Director of the Imaging Technology Center, FUJIFILM, for encouraging this research.

\section{G R A N T S}

This work was supported by a Grant-in-Aid for Japan Society for the Promotion of Science Fellows and Scientific Research (C), The Ministry of Education, Culture, Sports, Science and Technology, Japan (MEXT), and the Global Centers of Excellence Program "Revitalizing Education for Dynamic Hearts and Minds," MEXT, Japan.

\section{I S C L OS URES}

No conflicts of interest, financial or otherwise, are declared by the authors.

\section{REFERENCES}

Ban H, Yamamoto H, Fukunaga M, Nakagoshi A, Umeda M, Tanaka C, Ejima Y. Toward a common circle: interhemispheric contextual modulation in human early visual areas. J Neurosci 26: 8804-8809, 2006.

Bartels A, Zeki S. The architecture of the colour centre in the human visual brain: new results and a review. Eur J Neurosci 12: 172-193, 2000.

Becker MW, Anstis S. Metacontrast masking is specific to luminance polarity. Vision Res 44: 2537-2543, 2004.

Beer D, MacLeod DIA. Color selectivity in metacontrast: asymmetrical and anisotropic. J Vis 3: 138a, 2003.

Bevan W, Jonides J, Collyer SC. Chromatic relationships in metacontrast suppression. Psychonomic Sci 19: 367-368, 1970.

Boynton GM, Engel SA, Glover GH, Heeger DJ. Linear systems analysis of functional magnetic resonance imaging in human V1. J Neurosci 16: 4207-4221, 1996.

Brainard DH. Colorimetry. In: OSA Handbook of Optics: Fundamentals, Techniques, and Design, edited by Bass M. New York: McGraw-Hill, 1995, vol. 1 , chapt. 26 , p. $26.1-26.54$.

Breitmeyer BG, Ogmen H. Visual Masking: Time Slices Through Conscious And Unconscious Vision (2nd ed.). New York: Oxford, 2006.

Bridgeman B. Correlates of metacontrast in single cells of the cat visual system. Vision Res 15: 91-99, 1975.

Bridgeman B. Temporal response characteristics of cells in monkey striate cortex measured with metacontrast masking and brightness discrimination. Brain Res 196: 347-364, 1980.

Brockwell PJ, Davis RA. Time Series: Theory and Methods (2nd ed.). New York: Springer-Verlag, 1991, p. 14-25.

Brouwer GJ, Heeger DJ. Decoding and reconstructing color from responses in human visual cortex. J Neurosci 29: 13992-14003, 2009.
Cavonius CR, Reeves AJ. The interpretation of metacontrast and contrastflash spectral sensitivity functions. In: Colour Vision: Physiology and Psychophysics, edited by Mollon JD, Sharpe L. London: Academic Press, 1983, p. 471-478.

Cornelissen FW, Wade AR, Vladusich T, Dougherty RF, Wandell BA. No functional magnetic resonance imaging evidence for brightness and color filling-in in early human visual cortex. J Neurosci 26: 3634-3641, 2006.

Cowan W. Displays for vision research. In: OSA Handbook of Optics: Fundamentals, Techniques, and Design, edited by Bass M. New York: McGraw-Hill, 1995, vol. 1, chapt. 27 p. 27.1-27.44.

De Valois RL, Cottaris NP, Elfar SD, Mahon LE, Wilson JA. Some transformations of color information from lateral geniculate nucleus to striate cortex. Proc Natl Acad Sci USA 97: 4997-5002, 2000.

De Valois RL, De Valois KK. A multi-stage color model. Vision Res 33: 1053-1065, 1993.

Dijkstra EW. A note on two problems in connexion with graphs. Numerische Mathematik 1: 269-271, 1959.

Draper NR, Smith H. Applied Regression Analysis (3rd ed.). New York: John Wiley, 1998, p. 15-46.

Ejima Y, Takahashi S, Yamamoto H, Goda N. Visual perception of contextual effect and its neural correlates. In: Representation and Brain, edited by Funahashi S. Tokyo: Springer Verlag, 2007, p. 3-20.

Engel S, Zhang $\mathbf{X}$, Wandell B. Colour tuning in human visual cortex measured with functional magnetic resonance imaging. Nature 388: 68-71, 1997.

Engel SA, Rumelhart DE, Wandell BA, Lee AT, Glover GH, Chichilnisky EJ, Shadlen MN. fMRI of human visual cortex. Nature 369: 525, 1994.

Hanazawa A, Komatsu H, Murakami I. Neural selectivity for hue and saturation of colour in the primary visual cortex of the monkey. Eur $J$ Neurosci 12: 1753-1763, 2000.

Haynes JD, Driver J, Rees G. Visibility reflects dynamic changes of effective connectivity between V1 and fusiform cortex. Neuron 46: 811-821, 2005.

Huang J, Xiang M, Cao Y. Reduction in V1 activation associated with decreased visibility of a visual target. Neuroimage 31: 1693-1699, 2006.

Johnson EN, Hawken MJ, Shapley R. The spatial transformation of color in the primary visual cortex of the macaque monkey. Nat Neurosci 4: 409416, 2001.

Kiper DC, Fenstemaker SB, Gegenfurtner KR. Chromatic properties of neurons in macaque area V2. Vis Neurosci 14: 1061-1072, 1997.

Kolers PA, Green M. Color logic of apparent motion. Perception 13: 249-254, 1984.

Kondo H, Komatsu H. Suppression on neuronal responses by a metacontrast masking stimulus in monkey V4. Neurosci Res 36: 27-33, 2000.

Lennie P, Krauskopf J, Sclar G. Chromatic mechanisms in striate cortex of macaque. J Neurosci 10: 649-669, 1990.

Liu J, Wandell BA. Specializations for chromatic and temporal signals in human visual cortex. J Neurosci 25: 3459-3468, 2005.

Macknik SL. Visual masking approaches to visual awareness. Prog Brain Res 155: 177-215, 2006.

Macknik SL, Haglund MM. Optical images of visible and invisible percepts in the primary visual cortex of primates. Proc Natl Acad Sci USA 96: 15208-15210, 1999.

Macknik SL, Livingstone MS. Neuronal correlates of visibility and invisibility in the primate visual system. Nat Neurosci 1: 144-149, 1998.

MacLeod DI, Boynton RM. Chromaticity diagram showing cone excitation by stimuli of equal luminance. J Opt Soc Am 69: 1183-1186, 1979.

McKeefry DJ, Abdelaal S, Barrett BT, McGraw PV. Chromatic masking revealed by the standing wave of invisibility illusion. Perception 34: 913-920, 2005.

McKeown MJ, Makeig S, Brown GG, Jung TP, Kindermann SS, Bell AJ, Sejnowski TJ. Analysis of fMRI data by blind separation into independent spatial components. Hum Brain Mapp 6: 160-188, 1998.

Mullen KT, Dumoulin SO, Hess RF. Color responses of the human lateral geniculate nucleus: selective amplification of S-cone signals between the lateral geniculate nucleno and primary visual cortex measured with highfield fMRI. Eur J Neurosci 28: 1911-1923, 2008.

Mullen KT, Dumoulin SO, McMahon KL, de Zubicaray GI, Hess RF. Selectivity of human retinotopic visual cortex to S-cone-opponent, L/Mcone-opponent and achromatic stimulation. Eur J Neurosci 25: 491-502, 2007.

Ogawa S, Lee TM, Kay AR, Tank DW. Brain magnetic resonance imaging with contrast dependent on blood oxygenation. Proc Natl Acad Sci USA 87: 9868-9872, 1990. 
Ohtani Y, Okamura S, Yoshida Y, Toyama K, Ejima Y. Surround suppression in the human visual cortex: an analysis using magnetoencephalography. Vision Res 42: 1825-1835, 2002.

Otto TU, Ogmen H, Herzog MH. The flight path of the phoenix-the visible trace of invisible elements in human vision. J Vis 6: 1079-1086, 2006.

Parkes LM, Marsman JB, Oxley DC, Goulermas JY, Wuerger SM. Multivoxel fMRI analysis of color tuning in human primary visual cortex. $J$ Vis 9: 1.11079-13, 2009.

Ress D, Heeger DJ. Neuronal correlates of perception in early visual cortex. Nat Neurosci 6: 414-420, 2003.

Sankeralli MJ, Mullen KT. Bipolar or rectified chromatic detection mechanisms? Vis Neurosci 18: 127-135, 2001.

Sereno MI, Dale AM, Reppas JB, Kwong KK, Belliveau JW, Brady TJ, Rosen BR, Tootell RB. Borders of multiple visual areas in humans revealed by functional magnetic resonance imaging. Science 268: 889-893, 1995.

Smith VC, Pokorny J. Spectral sensitivity of the foveal cone photopigments between 400 and $500 \mathrm{~nm}$. Vision Res 15: 161-171, 1975.

Smith VC, Pokorny J. Color contrast under controlled chromatic adaptation reveals opponent rectification. Vision Res 36: 3087-3105, 1996.

Solomon SG, Lennie P. Chromatic gain controls in visual cortical neurons. $J$ Neurosci 25: 4779-4792, 2005.

Stoper AE, Banffy S. Relation of split apparent motion to metacontrast. J Exp Psychol Hum Percept Perform 3: 258-277, 1977.

Tootell RB, Tsao D, Vanduffel W. Neuroimaging weighs in: humans meet macaques in "primate" visual cortex. J Neurosci 23: 3981-3989, 2003.

Tse PU, Martinez Conde S, Schlegel AA, Macknik SL. Visibility, visual awareness, and visual masking of simple unattended targets are confined to areas in the occipital cortex beyond human V1/V2. Proc Natl Acad Sci USA 102: 17178-17183, 2005.

Uttal WR. On the physiological basis of masking with dotted visual noise. Percept Psychophys 7: 321-327, 1970.

von der Heydt R, Kaloudis P, Friedman H, Vemuri C. Metacontrast masking reveals narrowly tuned colour channels. Perception 27 (Suppl): 180,1998
Wachtler T, Sejnowski TJ, Albright TD. Representation of color stimuli in awake macaque primary visual cortex. Neuron 37: 681-691, 2003.

Wade A, Augath M, Logothetis N, Wandell B. fMRI measurements of color in macaque and human. J Vis 8: 6.1681-19, 2008.

Wandell BA, Baseler H, Poirson AB, Boynton GM, Engel S. Computational neuroimaging: color tuning in two human cortical areas measured using fMRI. In: Color Vision: From Genes to Perception, edited by Gegenfurtner KR, Sharpe LT. NewYork: Cambridge, 1999, p. 269-282.

Wandell BA, Chial S, Backus BT. Visualization and measurement of the cortical surface. J Cogn Neurosci 12: 739-752, 2000.

Williams AL, Singh KD, Smith AT. Surround modulation measured with functional MRI in the human visual cortex. J Neurophysiol 89: 525-533, 2003.

Woods RP, Grafton ST, Holmes CJ, Cherry SR, Mazziotta JC. Automated image registration. I. General methods and intrasubject, intramodality validation. J Comput Assist Tomogr 22: 139-152, 1998.

Worsley KJ, Friston KJ. Analysis of fMRI time-series revisited-again. Neuroimage 2: 173-181, 1995.

Xiao Y, Wang Y, Felleman DJ. A spatially organized representation of colour in macaque cortical area V2. Nature 421: 535-539, 2003.

Yamamoto H, Ban H, Fukunaga M, Umeda M, Tanaka C, Ejima Y. Large- and small-scale functional organization of visual field representation in the human visual cortex. In: Visual Cortex: New Research, edited by Portocello TA, Velloti RB. New York: Nova Science Publisher, 2008, p. 195-226.

Yamamoto H, Fukunaga M, Takahashi S, Tanaka C, Ebisu T, Umeda M, Ejima Y. BrainFactory: an integrated software system for surface-based analysis of fMRI data. Neuroimage 16: s443, 2002.

Yellott JI Jr, Wandell BA. Color properties of the contrast flash effect: monoptic vs dichoptic comparisons. Vision Res 16: 1275-1280, 1976.

Zenger-Landolt B, Heeger DJ. Response suppression in V1 agrees with psychophysics of surround masking. J Neurosci 23: 6884-6893, 2003. 